\title{
GASTOS EM SAÚDE E INCIDÊNCIA DE BENEFÍCIO NO MUNICÍPIO DE SENHOR DO BONFIM (BA) EM 2003*
}

\author{
Erika Aragão, Luciano Damasceno Santos ${ }^{*}$
}

Sebastião Loureiro ${ }^{* * *}$

Mônica Nunes ${ }^{* * *}$

\section{Isaura F. Gottschall de Almeida, Ricardo Luciano Silva Pereira de Souza ${ }^{* * * * *}$}

RESUMO O objetivo deste artigo é analisar a desigualdade no acesso e utilização dos serviços de saúde no município de Senhor do Bonfim (BA). O método utilizado é o "benefit incidence", ou incidência de benefício, que permite identificar quais os grupos sociais que se beneficiam dos gastos do Governo. A incidência de benefício no município de Senhor do Bonfim, para 2003, foi estimada por grupos da população previamente definidos (renda, gênero e cor da pele), com o objetivo de avaliar o grau de iniqüidade dos gastos públicos em saúde. Os gastos públicos foram desagregados por nível de complexidade. Combinando os dados referentes aos subsí-

* Artigo recebido em 2 de janeiro de 2006 e aprovado em 19 de março de 2007. Este artigo é resultado da pesquisa "As diferentes faces da desigualdade em saúde: iniqüidade, discriminação e exclusão", coordenada pelo professor Dr. Sebastião Loureiro, do Programa Economia da Saúde do Instituto de Saúde Coletiva da Bahia. A pesquisa foi financiada pelo DFID e teve apoio do Ministério da Saúde, IPEA e Secretaria Estadual da Saúde da Bahia.

** Mestres em economia pelo Curso de Mestrado em Economia da Universidade Federal da Bahia e pesquisadores do Programa Economia da Saúde do Instituto de Saúde Coletiva da UFBA. Erika Aragão também é professora das Faculdades Jorge Amado.

*** Doutor em Epidemiologia pela Universidade do Texas, mestre em Saúde Pública pela Universidade de Londres, médico pela Universidade Federal da Bahia.

$\star * \star *$ Professora do Instituto de Saúde Coletiva da UFBA, médica, mestre (UFBA), Ph.D. (Universidade de. Montreal).

***** Economistas pela FCE/UFBA e pesquisadores do PECS/ISC/UFBA. 
dios com os resultados da pesquisa domiciliar, observou-se iniqüidade da distribuição dos gastos públicos com saúde em Senhor do Bonfim.

Palavras-chave: desigualdade; acesso; serviços de saúde; Senhor do Bonfim; Bahia; incidência de benefício; gasto público

Código JEL: I180 - Health: Government Policy, regulation, public health; H510 national government expenditures and health

\section{PUBLICS EXPENDITURES AND BENEFIT INCIDENCE IN SENHOR DO BONFIM, CITY OF THE STATE OF BAHIA, IN 2003}

ABSTRACT The goal of this paper is to analyze inequality in the access and use of services of health in Senhor do Bonfim, city of the state of Bahia. It describes benefit incidence analysis, which allows to identify which social groups benefit governmental subsidies. The benefit incidence in Senhor do Bonfim, Bahia, was estimated, in 2003, for groups of the population previously defined (income, gender and race), with the objective of evaluating the inequity of the public expenditure in health in this city. The public expenses had been fragmented by complexity level. Combining the unit subsidies with the use patterns reported in the household survey reveals a very unequal distribution health spending in Senhor do Bonfim.

Key words: inequality; health services; benefit incidence; public expenditures; Senhor do Bonfim; Bahia 


\section{INTRODUÇÃO}

Este artigo é resultado de uma pesquisa intitulada "As diferentes faces da desigualdade em saúde: iniqüidade, discriminação e exclusão", que foi realizada a partir do convênio de cooperação técnica MS/IPEA/DFID. Esta pesquisa contém três níveis de análise. O primeiro utiliza os dados da Pesquisa Nacional por Amostragem Domiciliar - PNAD de 1998, para a Bahia. O segundo nível explora o método de benefit incidence para demonstrar iniqüidade na alocação de recursos públicos para a saúde. O terceiro nível utiliza um modelo de antropologia econômica em um corte intergeracional, de modo a revelar as mudanças no perfil da demanda, oferta e utilização, e concomitante, nas formas de financiamento de serviços de saúde, em três gerações de mulheres.

A relação entre nível de renda e saúde tem sido um dos temas mais estudados, tanto por economistas, quanto pesquisadores da área da Saúde Coletiva. As inúmeras pesquisas sobre esse tema abordam a relação entre o nível de renda e os vários aspectos da desigualdade em saúde, incluindo: renda e condição ou nível de saúde; renda e perfil de morbimortalidade; condição socioeconômica e padrão de mortalidade; nível de renda e demanda; acesso e utilização de serviços de saúde. Outra área de recente desenvolvimento são os estudos de desigualdade na alocação de recursos públicos para os serviços de saúde.

O método benefit incidence, ou incidência de benefício, tem sido muito utilizado em vários países para orientar políticas na alocação de recursos públicos para os segmentos mais carentes da população. ${ }^{1}$

A análise de incidência de benefícios situa-se no contexto da intervenção do Estado na provisão de bens públicos, para os quais o beneficio da coletividade é mais importante que o individual, tornando necessário corrigir, na medida do possível, as falhas de mercado existentes. A maior parte de sua aplicação concentra-se nos campos da educação e saúde, em função das externalidades positivas que o consumo desses bens promovem à sociedade, $\mathrm{O}$ que os caracterizam como bens de mérito.

Neste estudo foi feita uma aplicação do método benefit incidence, no município de Senhor do Bonfim, que no período de estudo (2004) era um dentre os 19 municípios da Bahia enquadrado como modelo de gestão ple- 
na do sistema municipal de saúde. Este trabalho está dividido em seis seções, considerando a introdução e as considerações finais. No sentido de apoiar a análise, sempre que necessário são utilizados resultados do estudo antropológico feito no município, também parte da pesquisa que resultou neste artigo.

\section{QUADRO TEÓRICO}

Várias pesquisas na área de economia e saúde coletiva sugerem que a utilização dos serviços de saúde é bastante desigual entre classes socioeconômicas, favorecendo as camadas mais privilegiadas da população. Pesquisadores como Wagstaff (2001) e Dachs (2001), têm explorado a construção de indicadores de desigualdade em saúde em relação à distribuição de renda nas populações. Uma revisão crítica da produção acadêmica latino-americana sobre desigualdade e saúde, a classificando as abordagens teóricas e os distintos conceitos presentes na literatura sobre desigualdade e saúde, pode ser encontrada em Almeida Filho (1999).

Dachs (2002) apresenta os fatores que explicam a desigualdade na autoavaliação do estado de saúde, mostrando que existem níveis de classificação do estado de saúde de acordo com a escolaridade, a renda, a raça e a cor da pele. Por meio de um modelo de regressão logística, o autor chega a resultados importantes dessa autoclassificação, percebendo que a educação e os rendimentos têm efeitos que se somam e que existem diferenças entre os homens e as mulheres.

Outro trabalho importante, com base nos dados da PNAD/SS-1998, que merece ser citado, é o de Sawyer et al. (2002). Nesse estudo sobre os perfis de utilização de serviços de saúde no Brasil, o autor identifica o padrão de consumo de serviços de saúde, por meio da técnica do Grade of Membership (GOM), que permite estimativas das demandas por serviços de saúde segundo níveis altos e baixos de capacitação, identificando quatro perfis de consumo de saúde, com destaque para o grupo de alta necessidade, predisposição e baixa capacitação, formado por pessoas idosas e que moram sozinhas.

Almeida et al. (2000), com base na Pesquisa Nacional sobre Saúde e Nutrição - PNSN, de 1989, constatam que a utilização dos serviços de saúde é bastante desigual entre as classes socioeconômicas, favorecendo as camadas 
de renda mais elevadas. Apenas cerca de 45\% dos indivíduos pertencentes ao primeiro quintil e que têm atividades restritas por motivo de doença utilizam os serviços de saúde. Esse percentual se eleva para 69,2\% quando são considerados os grupos de renda mais elevada.

Neri e Soares (2002) observam que os indivíduos nos primeiros decis da distribuição de renda têm pior acesso a seguro-saúde e, portanto, a despeito de necessitarem de maiores cuidados médicos, consomem menos os serviços de saúde.

Existe também estudo que compara a situação da saúde no Brasil com a saúde dos Estados Unidos da América. A comparação é feita tomando por base o grupo etário, sexo e raça ou cor da pele e as taxas de prevalência de morbidades selecionadas. Os principais resultados obtidos são que as mulheres se declaram em pior estado de saúde, em ambos os países, e os homens brasileiros se declaram em pior situação do que os homens norteamericanos (Beltrão, 2002).

Outra área de recente desenvolvimento são os estudos de desigualdade na alocação de recursos públicos para os serviços de saúde. Um método muito utilizado internacionalmente para subsidiar políticas públicas na alocação de recursos na área de saúde é o benefit incidence, ou incidência de benefício (Demery et al., 1995; Demery, 1996, 2000).

\section{O MODELO DE INCIDÊNCIA DE BENEFÍCIOS}

A análise de incidência de benefícios situa-se no contexto da intervenção do Estado na provisão de bens públicos, ${ }^{2}$ pois a atuação do governo é freqüentemente solicitada para subsidiar tais serviços, na medida em que o mercado não fornecerá ou o fará de forma insuficiente. Muitos desses bens são considerados bens de mérito, pois geram externalidades positivas. Portanto, para esses bens, o benefício da coletividade é mais importante que o individual.

Um importante ponto em relação à oferta desses bens é a distribuição dos benefícios ou gastos públicos para a população mais pobre, na medida em que muitas das políticas públicas têm por objetivo garantir aos grupos menos favorecidos o acesso aos bens e serviços básicos indispensáveis. Assim, monitorar a distribuição dos bens públicos é de extrema importância na condução de políticas que visem a minorar as desigualdades existentes. 
Este estudo procurou responder à seguinte questão: quem se beneficia dos recursos públicos destinados à saúde? Para respondê-la, foi realizada uma análise com base no método de incidência de benefícios. Esse método permite identificar quais os grupos sociais que mais se beneficiam dos subsídios governamentais, demonstrando se existe ou não iniqüidade na alocação de recursos públicos, neste caso, no setor de saúde.

A partir dessa perspectiva, decidiu-se analisar a incidência de benefício no município de Senhor do Bonfim, Estado da Bahia, que possui Gestão Plena do Sistema Municipal. Esta é uma aplicação inédita no Brasil utilizando dados primários. ${ }^{3}$ Uma adaptação do modelo no País foi feita por Diaz (2002) para dados da PNAD 1998. A maior parte dos demais estudos de incidência de benefício se deu no âmbito do Banco Mundial, sobretudo em países africanos e asiáticos (Demery et al., 1995; Demery, 1996; 2000; Castro-Leal et al., 1999).

Todos esses estudos apontam desigualdades na utilização dos recursos de saúde, desfavorecendo grupos de baixa renda. Ainda foram observadas desigualdades quando se consideram aspectos como gênero e raça/etnia.

Neste artigo, decidiu-se avaliar a população a partir dos seguintes critérios: renda, gênero e raça. A partir das variáveis selecionadas, foi possível identificar quais os grupos que mais se beneficiam dos gastos governamentais na oferta dos bens públicos. Para atingir esse objetivo, foram seguidos os passos da metodologia proposta por Demery (2000).

\subsection{Estimação da unidade de subsídio}

Refere-se à obtenção das estimativas do total de recurso para prover um serviço específico, neste caso, a saúde pública. Inicialmente, foram identificados os gastos públicos no setor e como eles são realizados. Para tanto, foram coletadas informações junto a fontes oficiais, como SIOPS, Datasus e contas governamentais e dos municípios, relativas ao segmento de saúde.

A análise de incidência de benefício deve considerar que o Brasil possui um sistema de financiamento do setor de saúde complexo, que busca responder às demandas dos níveis de atenção básico, médio e alto através de um sistema universal, unificado e descentralizado, segundo os princípios e diretrizes estabelecidos pela Constituição de 1988 e leis complementares emitidas, durante os anos 1990 com a sigla NOBs - Normas Operacionais 
Básicas, e, a partir de 2000, como NOAS - Normas Operacionais de Assistência à Saúde (Diaz, 2002).

As NOBs introduziram duas reformas de impacto no acesso à atenção básica: um pacote básico de serviços a serem providos pelos municípios à população e financiados pelo Governo Federal por um pagamento fixo per capita - PAB (Piso Assistencial Básico); e a responsabilização pela atenção de maior complexidade pelos municípios habilitados na "Gestão Plena do Sistema Municipal". O fluxo de recursos dentro do sistema de saúde depende do enquadramento do município aos tipos de gestão definidos na Norma Operacional Básica de 1996 (NOB-1996).

$\mathrm{Na}$ busca e seleção de dados junto a fontes governamentais, agruparamse os gastos do Governo por nível de complexidade. Como o município de Senhor do Bonfim dispõe apenas de serviços para os níveis de atenção de baixa e de média complexidade, as estimativas da utilização dos serviços de alta complexidade foram feitas a partir dos dados de uso dos serviços de alta complexidade pelos moradores desses municípios nos pólos que realizam esses procedimentos.

Relativamente aos recursos federais, o município de Senhor do Bonfim, por se enquadrar na Gestão Plena do Sistema Municipal, recebe os recursos destinados à saúde diretamente do Governo Federal. A União deposita o recurso diretamente nas contas da Prefeitura Municipal de Senhor do Bonfim. O município responsabiliza-se, conseqüentemente, pela assistência básica, de média e de alta complexidade existentes no município, além das ações vinculadas à Vigilância Sanitária. Entretanto, como não existe prestador de serviços de alta complexidade em Senhor do Bonfim, parte dos recursos destina-se a pagamentos de tratamento fora do domicílio (TFD).

Para os recursos federais e municipais, a fonte de informações básica é o SIOPS. No caso da média e alta complexidade (MAC), como o SIOPS apresenta informações agregadas, houve a necessidade de desagregá-las. Para tanto, foram utilizadas as proporções obtidas através de informações do Sistema de Informações Ambulatoriais - SIA/SUS e Sistemas de Informações Hospitalares - SIH/SUS. ${ }^{4}$

A distribuição dos atendimentos ambulatoriais e das internações entre diferentes níveis de complexidade deu-se mediante a classificação técnica dos procedimentos. A partir dos valores gastos nos procedimentos foi possível obter o percentual gasto com cada um dos níveis de complexidade. 
Tabela 1: Estrutura do gasto público em saúde em Senhor do Bonfim, 2003

\begin{tabular}{lcccccc}
\hline Nível de Atenção & \multicolumn{2}{c}{ Federal } & \multicolumn{2}{c}{ Municipal } & Total \\
& Valores (R\$) & $\%$ & Valores (R\$) & $\%$ & Valores (R\$) & $\%$ \\
\hline Assistência básica & $1.450 .128,95$ & 24,45 & $440.061,06$ & 24,4 & $1.890 .190,01$ & 24,4 \\
\hline Média complexidade & $3.854 .003,65$ & 64,97 & $1.359 .940,75$ & 75,6 & $5.213 .944,40$ & 67,4 \\
\hline Alta complexidade & $627.395,94$ & 10,58 & - & - & $627.395,94$ & 8,1 \\
\hline Total & 5.931.528,54 & 100,0 & $1.800 .001,81$ & 100,0 & $7.731 .530,35$ & 100,0 \\
\hline Fonte: Ministério da Saúde - (SIA/SUS), (SIH/SUS), SIOPS. & & & &
\end{tabular}

Para os gastos municipais, dado o caráter de custeio dos serviços de saúde (tais como pagamento de pessoal, energia, água, entre outros), os valores não estão definidos por nível de complexidade. Por essa razão, esses gastos foram distribuídos nos diferentes níveis, seguindo a mesma proporção observada nos gastos federais, apenas para atenção básica e média, já que o município não realiza procedimentos de alta complexidade.

O montante de gastos em saúde realizado pelas três esferas de Governo no município de Senhor do Bonfim em 2003, apresentado segundo a ótica do financiador, ou seja, pela origem dos recursos, soma $\mathrm{R} \$ 7,7$ milhões, conforme consta na tabela 1. Observa-se que não houve participação da esfera estadual, e que do total dos gastos a maior parte é federal (76\%), sendo o restante correspondente à esfera municipal.

Outro aspecto interessante a ser analisado refere-se à aplicação de recursos por nível de complexidade da atenção recebida pela população. Verificase que, de um total de aproximadamente $\mathrm{R}$ \$ 5,9 milhões que foram gastos em saúde no município de Senhor do Bonfim pelo Governo Federal, aproximadamente $24 \%$ destinaram-se à atenção básica, sendo $65 \%$ referentes à média e $10,6 \%$ à alta complexidade.

\subsection{Identificação dos usuários dos serviços}

Para identificar os usuários de serviços de saúde, foi realizada uma pesquisa de campo, dividida em duas etapas. Na primeira, foi escolhida uma amostra a partir dos dados disponíveis no Datasus para o município, por instituição de saúde. Os dados do Datasus indicaram um universo populacional para a atenção básica de 22.861, para média complexidade de 27.636 e para alta complexidade de 30. Daí calculou-se a amostra para os três níveis de atenção, com um índice de confiança de 90\%, conforme apresentado na tabe- 
la 2. Foi então aplicado um questionário nessas instituições buscando os dados dos pacientes para o mês selecionado (outubro de 2003). ${ }^{5} \mathrm{Na}$ segunda etapa, depois de definida a amostra e feita a coleta dos dados dos pacientes, foi realizada a aplicação dos questionários domiciliares, os quais totalizaram 310, aplicados na zona urbana da cidade de Senhor de Bonfim.

Para captar algum grau de exclusão, foram incorporadas questões como: se alguma vez o paciente já tinha deixado de utilizar o serviço público de saúde por algum tipo de restrição, dentre outras perguntas do gênero. Dessa maneira, a amostra incorpora os indivíduos sobre os quais incidiram os benefícios públicos, sem deixar de capturar aspectos relativos à exclusão.

Em muitos levantamentos, enfermidades e ferimentos, por exemplo, são auto-reportáveis. Isso pode causar um desvio nos dados se os respondentes, principalmente os mais humildes, encararem essas enfermidades e ferimentos como parte da vida comum e, portanto, não os reportarem.

Nos estudos efetuados pelo Banco Mundial, observou-se que as pessoas de baixa renda parecem fazer menos uso dos serviços, relativamente às que estão em melhores condições, simplesmente porque deixaram de identificar tal uso (não tinham conscientização disso).

No Vietnã, $74 \%$ das pessoas no quintil mais pobre (e apenas 55\% no quintil mais rico) reportaram uma enfermidade como autotratável ou que não precisava de tratamento. Os mais ricos apresentaram menor probabilidade de visitar uma clínica de serviços básicos do que os pobres (Demery, 2000).

Um segundo exemplo de desvios nos dados se baseia nas limitações da amostra utilizada para o levantamento. Esta geralmente não é estruturada para estimar eventos raros como visitas com internações no hospital. A estruturação do questionário desta pesquisa procurou introduzir questões que contemplassem essa dimensão, buscando, particularmente, evitar esse tipo de desvio.

Tabela 2: Total de atendimentos e amostra em outubro 2003 - Senhor do Bonfim

\begin{tabular}{lcccc}
\hline Tipo de Atenção & População & Amostra & Realizados & $\%$ \\
\hline Atenção básica & 22.861 & 74 & 104 & 140,5 \\
\hline Média complexidade & 27.636 & 206 & 191 & 92,7 \\
\hline Alta complexidade & 30 & 20 & 15 & 75,0 \\
\hline Total & 50.527 & 300 & 310 & 103,7 \\
\hline
\end{tabular}

Fonte: Nossos cálculos, a partir dos dados do Datasus - gerados através do programa Tabwin e pesquisa de campo. 


\subsection{Agregação dos indivíduos em grupos}

Com a disponibilidade dos dados das duas etapas anteriores, ou seja, com dados relativos aos gastos governamentais para os serviços e dados sobre a utilização desses serviços para a amostra populacional selecionada, agruparam-se os indivíduos pelas características previamente definidas: renda, gênero e raça.

Para mensurar a incidência de benefícios, é imprescindível que se obtenha o perfil socioeconômico das pessoas que utilizaram o serviço público de saúde. Essas características foram captadas nos questionários domiciliares, e os resultados permitem analisar, de forma mais geral, as classes que se beneficiam dos recursos públicos alocados em serviços de saúde na cidade de Senhor do Bonfim.

Recentes aplicações da metodologia aqui proposta demonstram o quanto esta pode ser uma boa ferramenta para se identificarem as desigualdades no acesso e na utilização dos serviços de saúde (Demery, 1996).

Castro-Leal et al. (1999) sistematizam estimativas da incidência do benefício de gastos públicos em saúde nos seguintes países da África: Costa do Marfim, Gana, Guiné, Quênia, Madagascar, África do Sul e Tanzânia. Os autores relatam que nesses sete países os pobres em geral foram menos beneficiados do que os grupos mais favorecidos.

Em uma aplicação em Gana, Demery (1996) demonstrou que a maior fonte de iniqüidade na distribuição dos benefícios dos gastos públicos em saúde é a dimensão de gênero. $\mathrm{O}$ estudo apontou que as mulheres recebem maiores parcelas dos benefícios dos subsídios (56\% dos gastos totais) em relação aos homens. Mas, pelo fato de as necessidades de saúde diferirem entre os sexos, isso ainda pode ser considerado um desvio no fornecimento dos serviços de saúde. Tal desvio pode ser mais bem observado analisandose o subgrupo sexo através dos quintis. As mulheres ganharam mais subsídios do que os homens nos dois quintis mais ricos dos serviços hospitalares como um todo. Para o restante da população, torna-se notório o desvio de recursos desfavorecendo as mulheres. Para os serviços hospitalares com internação, por exemplo, as mulheres ganharam apenas um terço do subsídio para o quintil.

Fora da África, os estudos também têm apontado desigualdades na utilização dos serviços de saúde. Na Bulgária, foi identificado que a taxa de uti- 
lização dos serviços de atenção básica pelos pobres é significativamente maior, enquanto os ricos utilizam os serviços mais complexos. Os turcos e ciganos na Bulgária são dois grupos minoritários no país, com cerca de 13\% do total da população. Esses grupos, entretanto, representam $25 \%$ do quintil mais pobre, e poucos deles são encontrados nos grupos de melhores condições. A disparidade racial no acesso ao serviço de saúde é verificada na medida em que um turco, na Bulgária, recebeu subsídio de apenas 1.001 leva (moeda da Bulgária) e um cigano, 1.446 leva. Já os indivíduos do principal grupo étnico da Bulgária receberam 2.802 leva per capita. Os turcos e ciganos representam, portanto, $13 \%$ da população, mas receberam apenas $6 \%$ do subsídio da saúde (Demery, 2000).

Em sua análise dos dados da PNAD 1998, Diaz (2002) observou que os indivíduos do primeiro quintil de renda se apropriaram de um percentual inferior dos recursos públicos destinados à atenção básica. No que tange à média e alta complexidade, o primeiro quintil só não se apropriou de menos recursos que os indivíduos do quinto quintil, ou seja, aqueles relativamente mais ricos.

A análise da incidência do beneficio possibilita, assim, a discriminação da iniqüidade na utilização dos serviços de saúde associada com: (a) subutilização generalizada dos serviços; (b) utilização desproporcional por grupos carentes. A análise dos impactos dos gastos públicos com saúde pode, portanto, subsidiar avaliações do grau de cumprimento de metas de redução das desigualdades existentes.

\section{RESULTADOS DA PESQUISA DE CAMPO}

\subsection{Perfil socioeconômico dos pacientes}

Neste estudo, as informações demográficas da pesquisa de campo (questionários domiciliares) foram comparadas com os dados do Censo Demográfico 2000 - IBGE, para o município de Senhor do Bonfim. A comparação entre os dados do estudo e da população recenseada permite precisar melhor as características dos pacientes.

Segundo a pesquisa, cerca de dois terços dos pacientes inquiridos são do sexo feminino $(67,2 \%)$. A proporção maior no sexo feminino indica que as mulheres utilizam com maior freqüência os serviços de saúde comparativa- 
mente ao sexo masculino, ainda que a proporção de homens $(48,6 \%)$ e mulheres $(51,4 \%)$ na população seja praticamente igual, com uma leve maioria de mulheres. ${ }^{6}$

Em Senhor do Bonfim, a utilização do serviço público de saúde apresenta-se mais concentrada entre os 20 e 39 anos de idade. Dados que há uma concentração na utilização do serviço na faixa de 20 a 39 anos em Senhor de Bonfim e que a proporção de mulheres utilizando o serviço é superior à dos homens, confirma-se que essa utilização está ligada estreitamente à idade fértil das mulheres. Mas não só a esse fator.

Os resultados de uma análise antropológica da pesquisa "As diferentes faces da desigualdade em saúde: iniqüidade, discriminação e exclusão", da qual resultou este artigo, trazem dimensões mais subjetivas, relativas a questões culturais: na nossa sociedade, o fato de a mulher ser orientada a um maior cuidado com o corpo torna a prevenção uma característica mais feminina; os homens, por sua vez, em função do culto à virilidade, minimizam as questões corporais e tendem a desenvolver uma atitude defensiva, que parece ser uma expressão do medo de tratar-se, o que faz com que recorram mais tardiamente ao sistema de saúde.

No que diz respeito ao grau de instrução, observou-se que o percentual de pacientes com até primeiro grau incompleto é de 65,2\%, inferior aos $78,7 \%$ da população segundo o senso de 2000 . Vale chamar a atenção para o percentual de pacientes na categoria sem escolaridade/analfabetos $(24,1 \%)$, o qual permanece elevado mesmo considerando o número de pacientes com menos de 10 anos (13,2\%), dos quais muitos não estão em idade escolar e incorporam a categoria sem escolaridade/analfabetos.

No outro extremo, a pesquisa de campo praticamente não registrou indivíduos com instrução superior, percebendo apenas um indivíduo para a cidade, o que representa 0,3\% em Senhor do Bonfim. Contudo, tais dados devem ser interpretados com cuidado, pois esses números não representam o perfil da população desse município, mas apenas o perfil específico da clientela que utilizou os recursos do SUS. Os dados do censo 2000 mostram que 2,3\% da população de Senhor do Bonfim possuem nível superior.

Quanto à situação ocupacional, predominaram os ocupados, os estudantes e as donas de casa, sendo o percentual de desempregados que faz uso do serviço bastante baixo $(8,93 \%)$. Uma das explicações para esse quadro é 
o fato de que os custos com transporte são significativos em função das distâncias em Senhor do Bonfim serem consideravelmente grandes, questão essa ressaltada pelos entrevistados.

Um dos aspectos mais importantes do estudo é a análise da renda das famílias dos pacientes, e, para tanto, optou-se em dividir a amostra em quintis, ou seja, cada uma das cinco partes possui cerca de $20 \%$ da amostra dos pacientes. A renda utilizada foi a renda total do domicílio dividida pelo número de moradores do mesmo, obtendo a renda familiar per capita.

Dos pacientes do SUS em Senhor do Bonfim, apenas 20\% percebem uma renda per capita acima de $\mathrm{R} \$ 192,3$, rendimento inferior a um salário mínimo. Para complementar a análise da renda, elaborou-se faixas de renda familiar per capita (percentuais do salário mínimo).

Em Senhor do Bonfim, a maior concentração encontra-se na primeira faixa (até $1 / 4$ de salário mínimo), com 35,16\%. Em seguida vem a segunda faixa, com $33,87 \%$ da população pesquisada. A menor concentração está na faixa acima de um salário mínimo (8,39\%). Quando somadas as duas primeiras faixas, percebe-se a grande concentração de pacientes nas faixas de mais baixo poder aquisitivo, $69,03 \%$.

Tabela 3: Quintis de renda familiar per capita

\begin{tabular}{ll}
\hline Quintil de renda per capita & R\$ \\
\hline $1^{\circ}$ Quintil & até 52 \\
\hline $2^{\circ}$ Quintil & acima de 52,0 a 75,0 \\
\hline $3^{\circ}$ Quintil & acima de 75,0 a 117,3 \\
\hline $4^{\circ}$ Quintil & acima de 117,3 a 192,3 \\
\hline $5^{\circ}$ Quintil & acima de 192,3 \\
\hline Fonte: Pesquisa de campo. &
\end{tabular}

Tabela 4: Faixas de renda familiar per capita

\begin{tabular}{lccc}
\hline & $n^{\circ}$ & Senhor do Bonfim & \\
\hline Até $1 / 4$ SM & 109 & 35,16 & 35,16 \\
\hline De $1 / 4$ a $1 / 2$ SM & 105 & 33,87 & 69,03 \\
\hline De $1 / 2$ a $3 / 4$ SM & 40 & 12,9 & 81,93 \\
\hline De $3 / 4$ a 1 SM & 30 & 9,68 & 91,61 \\
\hline Acima de 1 SM & 26 & 8,39 & 100,0 \\
\hline Total & 310 & 100,0 & \\
\hline
\end{tabular}

Fonte: Pesquisa de campo. 


\subsection{Condições de saneamento}

Entre os problemas que comprometem o desenvolvimento no Brasil e no resto do mundo está a questão do saneamento básico, que está associado intimamente ao padrão socioeconômico da população. Falta de acesso, ou acesso precário a saneamento básico comprometem a saúde, afetando o trabalho e a produtividade dos indivíduos e da economia. Estudos do Banco Mundial mostram que cada dólar que se investe em saneamento permite que se deixe de gastar quatro dólares em Saúde (Furtado, 2004).

Em Senhor do Bonfim, da amostra selecionada, 98,7\% dos usuários dos serviços de saúde da cidade disseram possuir água canalizada. Nesse município, em 97,7\% dos casos, a água é proveniente de rede geral de distribuição. Se perguntados se dispõem de banheiro ou sanitário, 97,7\% dos indivíduos de Senhor do Bonfim responderam sim. No que se refere à forma de escoamento dos resíduos provenientes dessas instalações, $67,8 \%$ dos entrevistados nesse município relataram a rede de coleta de esgoto, e 29,6\% afirmaram utilizar a fossa séptica.

O Censo de 2000 do IBGE mostra que, no Brasil, $89,9 \%$ dos domicílios da zona urbana dispunham de serviço de água, contra 18,7\% da zona rural. No que tange à rede de esgotamento, 56\% dos domicílios da zona urbana tinham cobertura, contra apenas $3,3 \%$ da zona rural. A cobertura por via da fossa séptica é de respectivamente $16 \%$ e 9,6\%.

Tabela 5: Condições de saneamento (\%)

\begin{tabular}{lc}
\hline Condições de Saneamento & \multicolumn{1}{c}{ Senhor do Bonfim } \\
\hline Água canalizada & 98,7 \\
\hline Água proveniente de: & 97,7 \\
Rede geral & 0,3 \\
\hline Poço & 1,9 \\
\hline Outra forma & 97,7 \\
\hline Banheiro ou sanitário & \\
\hline Forma de coleta do esgoto: & 67,8 \\
\hline Rede de coleta de esgoto & 29,6 \\
\hline Fossa séptica & 0,0 \\
\hline Fossa rudimentar & 1,0 \\
\hline Vala & 1,6 \\
\hline Outra forma &
\end{tabular}




\subsection{Estado de saúde}

Em geral, existe consenso de que as condições socioeconômicas são determinantes no que se refere ao estado de saúde dos indivíduos, como podemos verificar, por exemplo, em Diaz (2001); House, Kesseler e Regula (1990); Noronha et al. (2002a); Noronha et al. (2002b); Viana, Fausto e Lima (2003); Wood e Carvalho (1994); Travassos et al. (2000), além dos já citados estudos feitos no âmbito do Banco Mundial. Se uma pessoa perde o emprego, ou seu nível de renda é muito baixo, ela se alimenta mal, comprometendo sua saúde, e, quando adoece, muitas vezes não tem recursos nem para procurar o médico. Caso consiga assistência, não dispõe de recursos para comprar medicamentos. O nível de escolaridade também afeta as condições de saúde. Além disso, observa-se a relação entre baixa escolaridade da mãe e mortalidade infantil, como constata Viana et al. (2001).

Mas, além das questões socioeconômicas e de saneamento básico, vários estudos apontam que condições de saúde também estão associadas ao gênero e à raça dos indivíduos, seja por aspectos biológicos, como a idade fértil das mulheres, culturais, ou por motivo de discriminação no que tange à cor da pele, o que é muito difícil de ser caracterizado (Oliveira, 1995; Oliveira, 2001; Travassos et al., 2001; Demery et al., 1995; Demery, 1996, 2000; Castro-Leal et al., 1999). Assim, não surpreende que, quando são avaliadas questões referentes ao estado de saúde dos indivíduos em Senhor do Bonfim, se observam diferenças razoáveis quando são incorporados esses aspectos.

Em seu conjunto, 43,5\% dos entrevistados em Senhor do Bonfim alegaram bom estado de saúde, contra 13,2\%, que alegaram estado ruim. Outros 43,2\% alegaram estado de saúde regular.

Porém, quando se introduz o fator renda, observa-se que os maiores percentuais de pessoas que identificam seu estado de saúde como ruim estão no primeiro quintil: 19,2\%. No quinto quintil, esse percentual é de apenas 10\%. Parece haver em Senhor do Bonfim uma tendência significativa de queda na proporção de pessoas que avaliam negativamente sua situação de saúde, à medida que se eleva o nível de renda. Esse é um fato curioso, na medida em que, em muitos levantamentos, como aplicações de incidência de benefício feitas por Demery (2000), a população mais humilde tende a encarar essas 
Tabela 6: Estado de saúde por quintil de renda (\%)

\begin{tabular}{lrrrrr}
\hline Estado de Saúde & \multicolumn{5}{c}{ Senhor do Bonfim } \\
\hline & 1 & 2 & 3 & 4 & 5 \\
\hline Bom & 46,6 & 46,2 & 42,9 & 40,7 & 43,3 \\
\hline Regular & 34,2 & 46,2 & 44,6 & 45,8 & 46,7 \\
\hline Ruim & 19,2 & 7,7 & 12,5 & 13,6 & 10,0 \\
\hline Total & 100,0 & 100,0 & 100,0 & 100,0 & 100,0 \\
\hline Fonte: Pesquisa de campo. & & & & &
\end{tabular}

Tabela 7: Estado de saúde por sexo (\%)

\begin{tabular}{lcc}
\hline Estado de Saúde & \multicolumn{2}{c}{ Senhor do Bonfim } \\
\hline & Masculino & Feminino \\
\hline Bom & 41,6 & 44,5 \\
\hline Regular & 47,5 & 41,1 \\
\hline Ruim & 10,9 & 14,4 \\
\hline Total & 100,0 & 100,0 \\
\hline
\end{tabular}

Fonte: Pesquisa de campo.

enfermidades e ferimentos como parte da vida comum e, portanto, não os reportam.

Em uma análise dos dados da PNAD, o resultado encontrado também aponta em outra direção: $85 \%$ das pessoas no quintil de renda mais baixo se auto-avaliam no estado de saúde na categoria bom, caindo até $72,8 \%$ no quarto quintil e chegando a $80 \%$ das pessoas no último quintil. Enquanto isso, a porcentagem das pessoas que se classificam com saúde ruim sobe de $2,4 \%$ a 3,6\%, ou seja, quanto maior o nível de renda pior é a autopercepção do estado de saúde.

Quando se segmenta a amostra por cor da pele, verifica-se que, em Senhor do Bonfim, os brancos, em geral, reportam melhor estado de saúde que os negros: $44,3 \%$ dos brancos e $43,2 \%$ dos negros reportam como bom o seu estado de saúde. Por sua vez, respectivamente $10,2 \%$ e $14,4 \%$ alegam ruim a sua condição de saúde.

Quando se observa a percepção do estado de saúde por gênero, verificase que, em Senhor do Bonfim, o percentual de mulheres (44,5\%) que têm uma boa percepção de seu estado de saúde é ligeiramente maior que o de homens $(41,6 \%)$.

Dachs (2002) apresenta um estudo que busca definir os determinantes na explicação das desigualdades na auto-avaliação do estado de saúde. Uti- 
lizando um modelo de regressão logística, constata que a idade é a mais importante variável de autopercepção do estado de saúde, e quanto menor a idade, melhor a avaliação da saúde. A auto-avaliação do estado de saúde como "muito bom" está relacionada positivamente, também, com o aumento nos níveis de educação e rendimentos, enquanto a probabilidade de estar classificada como uma saúde boa é menor para as mulheres. Estas duas últimas correlações não foram observadas em Senhor do Bonfim.

\subsection{Acesso aos serviços de saúde}

No que se refere ao acesso aos serviços de saúde, quando perguntados se possuem plano de saúde, $82 \%$ dos entrevistados em Senhor do Bonfim responderam não. Esse fato não surpreende, considerando-se o perfil da amostra - usuários do serviço público de saúde - e os quintis de renda. A porcentagem relativamente grande de usuários que possui plano ou seguro-saúde (18\%) pode ser explicada em função da existência de planos de saúde, ligados a associações, que têm cobertura limitada, ou seja, quando aparecem procedimentos não cobertos pelo plano esses usuários procuram o sistema público de saúde.

No Brasil, a universalização da saúde pública, instituída como princípio constitucional em 1988, aconteceu associada à ampliação do sistema privado de saúde, para onde migrou parcela significativa da classe média, que antes tinha acesso ao sistema mediante contribuição previdenciária. Nesse processo, uma modalidade muito comum de seguro privado à qual parcelas mais pobres da população também passaram a ter acesso é a empresarial. Na maior parte dos casos, as empresas arcam com planos ou seguros de saúde com cobertura limitada e, caso os funcionários queiram ampliar essa cobertura, têm de pagar a diferença. Isso normalmente não é feito pelos indivíduos com remuneração mais baixa, que ficam com a cobertura básica paga pelas empresas. Logo, procedimentos não cobertos são realizados no âmbito do SUS.

A renda é um fator muito importante, pois, no que tange à posse de plano de saúde, implica uma distribuição diferenciada por quintil de renda: $6,8 \%$ dos pertencentes ao primeiro quintil alegaram possuir plano de saúde, contra respectivamente $28,3 \%$ do quinto quintil. 
Tabela 8: Plano de saúde por quintil de renda

\begin{tabular}{|c|c|c|}
\hline \multicolumn{3}{|c|}{ Senhor do Bonfim } \\
\hline Quintis & Sim & Não \\
\hline $1^{\circ}$ Quintil & $6,80 \%$ & $93,20 \%$ \\
\hline $2^{\circ}$ Quintil & $13,50 \%$ & $86,50 \%$ \\
\hline $3^{\circ}$ Quintil & $23,20 \%$ & $76,80 \%$ \\
\hline $4^{\circ}$ Quintil & $20,00 \%$ & $80,00 \%$ \\
\hline $5^{\circ}$ Quintil & $28,30 \%$ & $71,70 \%$ \\
\hline Total & $17,90 \%$ & $82,10 \%$ \\
\hline
\end{tabular}

Em Senhor do Bonfim, cerca de 90\% dos entrevistados costumam procurar o mesmo lugar quando precisam de atendimento. Um fator que pode contribuir para isso é a organização do sistema de saúde nesse município, que, por estar enquadrado como gestão plena do sistema municipal, possui um sistema de saúde mais organizado, contando, inclusive, com uma central de marcação de consultas para os postos de saúde, o que assegura, por exemplo, menos tempo de espera para o atendimento.

Apesar das críticas feitas pelos usuários captadas pelo estudo antropológico realizado na pesquisa da qual resultou este artigo, em geral, os níveis de satisfação com os serviços de saúde são consideráveis em Senhor do Bonfim. Porém, 89,40\% dos entrevistados não foram atendidos na primeira vez da procura, a maioria alegando, como motivo do não-atendimento, não haver conseguido vaga. Sobre isso, o estudo antropológico realizado aponta que a percepção dos usuários é que isso ocorre por ineficiência da central de marcação.

Quando está doente, a maior parte dos usuários da rede pública de saúde em Senhor do Bonfim (66,9\%) procura postos ou centros de saúde. Isso se explica pelo fato de que nesse município existem mais postos de saúde, e o acesso para os seus serviços através da central de marcação é facilitado. O acesso a esses serviços se deu através de marcação (66,6\%). Outros 29,9\% dos atendimentos foram de urgência, e apenas 1,3\% facilitado por agentes comunitários.

Um percentual significativo daqueles que procuraram atendimento médico em outubro de 2003 deixou de realizar alguma atividade em função do problema de saúde relacionado: 30,9\%. Observou-se que o adoecimento e o tratamento afastam o indivíduo de atividades remuneradas, sobretudo os 
que não possuem emprego formal, e de outras não remuneradas, mas de extrema importância, como tomar conta dos filhos pequenos.

Os estudos de incidência de benefício aqui listados (Demery et al., 1995, Demery, 1996, 2000; Castro-Leal et al., 1999) mostram que os custos diretos (com remédios, transporte etc.) e indiretos (babá, acompanhante, custo de oportunidade de não estar fazendo outra atividade) pesam significativamente no tratamento. Nesse caso, aqueles que possuem uma rede social de apoio tendem a ser mais bem-sucedidos no tratamento.

$\mathrm{O}$ acesso a medicamento gratuito distribuído pelo SUS por seus diversos programas é um fator importante e, em muitos casos, determinante para o tratamento, sobretudo no que tange aos medicamentos de alto custo, tendo em vista o baixo rendimento da população. Nesse quesito, observou-se que 36,7\% dos usuários do sistema público entrevistados não receberam remédio gratuito. Isso preocupa, visto que em Senhor do Bonfim os usuários do SUS apresentam um rendimento muito baixo, como mostram as tabelas $3 \mathrm{e}$ 4. Porém, vale destacar que os percentuais daqueles que não sabem ou não lembram é significativo, o que pode estar influenciando os resultados.

No que se refere à resolubilidade do tratamento, 25,4\% dos usuários do SUS alegaram que o atendimento recebido não resolveu o problema. A es-

Tabela 9: Deixou de procurar o serviço de saúde por alguma restrição

\begin{tabular}{lc}
\hline Restrição & Senhor do Bonfim \\
\hline Não tinha com quem deixar os filhos & $3,20 \%$ \\
\hline Não tinha dinheiro & $35,50 \%$ \\
\hline Local de atendimento era distante ou de difícil acesso & $1,60 \%$ \\
\hline Dificuldade de transporte (falta) & $6,50 \%$ \\
\hline Horário incompatível & $30,60 \%$ \\
\hline Atendimento é muito demorado & $4,80 \%$ \\
\hline Não possuía especialista compatível com as necessidades & $9,70 \%$ \\
\hline Não sabe ou não lembra & $1,60 \%$ \\
\hline Outro motivo & \\
\hline Esqueceu de ir & $3,20 \%$ \\
Não quer se submeter ao tratamento & $1,60 \%$ \\
\hline Não tinha vaga & $1,60 \%$ \\
\hline Só vai em último caso & $100,00 \%$ \\
\hline Total & \\
\hline Fonte: Pesquisa de campo. & \\
\hline
\end{tabular}


cassez de profissionais, a não-disponibilidade, ou a disponibilidade restrita a medicamentos, a carência de exames complementares, dentre outros fatores, contribuem para essas elevadas taxas.

Quando questionados acerca da existência de alguma restrição que tenha impedido de procurar o serviço de saúde, os motivos mais citados foram a falta de dinheiro, alcançando um percentual de mais de 35\%, e a demora no atendimento, chamando a atenção o percentual de 30,6\%.

\section{APLICAÇÃO DO MÉTOdo INCIDÊNCIA DE BENEFÍCIOS EM SENHOR DO BONFIM}

Depois de mapeado o perfil da população dos usuários do sistema público de saúde em Senhor do Bonfim, foi realizada a análise de incidência de benefícios, tendo por objetivo avaliar a incidência dos gastos públicos sobre os diferentes grupos da população, especialmente os mais pobres. Essa análise vem subsidiar avaliações do grau de redução da pobreza e de iniqüidades.

A análise de incidência do gasto público em saúde no município de Senhor do Bonfim permite concluir que os indivíduos pertencentes ao primeiro quintil da distribuição beneficiaram-se mais dos gastos públicos em saúde em atenção básica do que os pertencentes aos demais quintis. Enquanto os indivíduos pertencentes ao primeiro quintil se apropriaram de 28,7\% dos recursos públicos destinados à atenção básica, os indivíduos do último quintil, ou seja, os mais ricos se apropriaram de 17,8\% dos recursos públicos destinados à saúde nesse município no ano 2003.

Com relação à atenção de média complexidade, não foram observadas diferenças significativas entre os percentuais apropriados pelos indivíduos pertencentes aos quintis. Apenas para os indivíduos do segundo quintil o percentual de apropriação dos recursos foi de 15,6\%; para os demais quintis, ficou em torno de $20 \%$.

Os dados relativos à atenção de alta complexidade merecem alguns cuidados, pois o número de observações presentes na pesquisa foi de apenas 15, o que tem implicações sobre a efetiva representatividade dos resultados obtidos acerca desse nível de complexidade. Levando em consideração tal problema, os dados sugerem que os indivíduos pertencentes ao quinto quintil, ou seja, os 20\% mais ricos da distribuição, se apropriaram de 33,3\% 
Tabela 10: Percentual de apropriação dos recursos públicos segundo o nível de assistência por quintil de renda em Senhor do Bonfim, 2003

\begin{tabular}{|c|c|c|c|c|c|c|}
\hline Nível de Atenção & 1 & 2 & 3 & 4 & 5 & Total \\
\hline $\begin{array}{l}\text { Assistência } \\
\text { básica }\end{array}$ & $542.727,82$ & $374.295,05$ & $280.721,29$ & $355.580,30$ & $336.865,55$ & $1.890 .190,01$ \\
\hline $\begin{array}{l}\text { Percentual } \\
\text { de apropriação }\end{array}$ & 28,7 & 19,8 & 14,9 & 18,8 & 17,8 & 100,0 \\
\hline $\begin{array}{l}\text { Média } \\
\text { complexidade }\end{array}$ & $1.177 .342,28$ & $812.926,82$ & $1.121 .278,37$ & $1.065 .214,45$ & $1.037 .182,49$ & $5.213 .944,40$ \\
\hline $\begin{array}{l}\text { Percentual } \\
\text { de apropriação }\end{array}$ & 22,6 & 15,6 & 21,5 & 20,4 & 19,9 & 100,0 \\
\hline $\begin{array}{l}\text { Alta } \\
\text { complexidade }\end{array}$ & $83.652,79$ & $125.479,19$ & $83.652,79$ & $125.479,19$ & $209.131,98$ & $627.395,94$ \\
\hline $\begin{array}{l}\text { Percentual } \\
\text { de apropriação }\end{array}$ & 13,3 & 20,0 & 13,3 & 20,0 & 33,3 & 100,0 \\
\hline Total geral & $1.868 .879,85$ & $1.331 .256,88$ & $1.459 .262,35$ & $1.536 .065,63$ & $1.536 .065,63$ & $7.731 .530,35$ \\
\hline $\begin{array}{l}\text { Percentual } \\
\text { de apropriação }\end{array}$ & 24,2 & 17,2 & 18,9 & 19,9 & 19,9 & 100,0 \\
\hline
\end{tabular}

dos recursos públicos destinados a esse nível de atenção, enquanto os indivíduos pertencentes ao primeiro quintil se apropriaram apenas de 13,3\% dos recursos.

Quando se analisa a apropriação do gasto público em saúde por gênero, verifica-se uma diferença considerável entre os percentuais de apropriação dos recursos públicos gastos em saúde entre homens e mulheres nos diferentes níveis de complexidade da atenção. As mulheres se apropriam de $71,2 \%$ e 66,7\% dos recursos destinados à atenção básica e de média complexidade, respectivamente; percentuais bem superiores aos dos homens. Já no nível de atenção de alta complexidade, os resultados alteram-se, com os homens apropriando-se de 53,3\% dos recursos destinados a esse nível de atenção. Como já foi mencionado, isso se deve à idade fértil das mulheres e a questões tipicamente culturais.

Demery (2000) obteve um resultado semelhante na aplicação do modelo em Gana: as mulheres, no total, ganharam mais subsídios na saúde do que os homens (56\% do gasto total em 1992 - ver Benefit incidence: a practicioner's guide, tabela 16, p. 35). Mas, como foi mencionado anteriormente, o fato de as necessidades de saúde diferirem entre os sexos ainda pode ser considerado um desvio no fornecimento dos serviços de saúde. 
Tabela 11: Percentual de apropriação dos recursos públicos segundo o sexo em Senhor do Bonfim, 2003

\begin{tabular}{lccc}
\hline Nível de Atenção & Masculino & Feminino & Total \\
\hline Assistência básica & $571.002,50$ & $1.408 .472,82$ & $1.979 .475,32$ \\
\hline Percentual de apropriação & 28,8 & 71,2 & 100,0 \\
\hline Média complexidade & $1.749 .087,56$ & $3.498 .175,11$ & $5.247 .262,67$ \\
\hline Percentual de apropriação & 33,3 & 66,7 & 100,0 \\
\hline Alta complexidade & $365.033,58$ & $319.404,39$ & $684.437,97$ \\
\hline Percentual de apropriação & 53,3 & 46,7 & 100,0 \\
\hline Total geral & $2.594 .662,21$ & $5.316 .513,75$ & $7.911 .175,96$ \\
\hline Percentual de apropriação & 32,8 & 67,2 & 100,0 \\
\hline
\end{tabular}

Fonte: Elaboração própria a partir da pesquisa de campo e dados do Datasus.

Tabela 12: Percentual de apropriação dos recursos públicos segundo a cor da pele em Senhor do Bonfim, 2003

\begin{tabular}{lccc}
\hline Nível de Atenção & Brancos & Negros & Total \\
\hline Assistência básica & $599.771,83$ & $1.290 .418,18$ & $1.890 .190,01$ \\
\hline Percentual de apropriação & 31,7 & 68,3 & 100,0 \\
\hline Média complexidade & $1.412 .109,94$ & $3.801 .834,46$ & $5.213 .944,40$ \\
\hline Percentual de apropriação & 27,1 & 72,9 & 100,0 \\
\hline Alta complexidade & $167.305,58$ & $460.090,36$ & $627.395,94$ \\
\hline Percentual de apropriação & 26,7 & 73,3 & 100,0 \\
\hline Total geral & $2.212 .560,13$ & $5.518 .970,22$ & $7.731 .530,35$ \\
\hline Percentual de apropriação & 28,6 & 71,4 & 100,0 \\
\hline
\end{tabular}

Fonte: Elaboração própria a partir da pesquisa de campo e dados do Datasus.

No que tange à cor da pele, pode-se verificar que os negros são predominantes na apropriação dos recursos públicos destinados à saúde em todos os níveis de complexidade. No total geral, os brancos se apropriaram de 28,6\%, e os negros, de 71,4\% desses recursos. Vale destacar, mais uma vez, a agregação: os negros e os pardos foram considerados negros.

\section{CONSIDERAÇÕES FINAIS}

A pesquisa de campo e a análise de incidência de benefícios revelaram elementos de extrema relevância quanto ao acesso e à utilização dos serviços públicos de saúde no município de Senhor do Bonfim, permitindo identificar iniqüidades, tanto no âmbito do enfoque descritivo quanto no da incidência propriamente dita. 
A análise do gasto público seguiu na direção de procurar desagregar o gasto público por nível de complexidade do atendimento. Verificou-se, em 2003, que o montante de gastos em saúde realizado pelas esferas de Governo no município de Senhor do Bonfim foi de R\$7.731.530, não tendo havido participação da esfera estadual. Verifica-se que a maior parte do gasto é federal, alcançando $76 \%$ do total.

No que diz respeito à apropriação dos gastos pela população, considerando-se os grupos propostos, observou-se que as mulheres se apropriaram da maior parte do total dos recursos $(67,2 \%)$, ou seja, em torno do dobro dos homens. Quando se faz o recorte por nível de atenção, contudo, verificase que as mulheres se apropriam relativamente menos dos recursos de alta complexidade.

Quando se faz o recorte por renda, observa-se, no geral, que os indivíduos dos quintis de renda superiores se apropriam da maior parte dos recursos públicos destinados à alta complexidade. Esse resultado aponta que os grupos relativamente "mais ricos" estão se apropriando dos procedimentos mais caros. Os resultados das entrevistas mostram que o fato de que os mais ricos estão se apropriando dos procedimentos mais caros se dá tanto em função de maior informação do grupo relativamente mais rico quanto pelo maior capital social desse grupo.

Fatores como indicações de amigos e parentes que trabalham ou conhecem alguém para conseguir realizar determinado procedimento e obter remédios e transporte gratuitos foram apontados recorrentemente. A procura por parte de indivíduos "mais ricos" de serviços de alta complexidade na rede pública é compreensível até para níveis bem elevados de renda, pois muitos desses procedimentos são de alto custo e os medicamentos utilizados no tratamento são muito caros, não sendo cobertos por muitos planos de saúde privados.

Os indivíduos pertencentes ao primeiro quintil da distribuição beneficiaram-se mais dos gastos públicos em saúde em atenção básica do que os pertencentes aos demais quintis, em consonância com os resultados encontrados na literatura. Com relação à atenção de média complexidade, não foram observadas diferenças significativas entre os percentuais apropriados pelos indivíduos pertencentes aos quintis. 
$\mathrm{Na}$ análise por cor da pele, observou-se que os negros se apropriam da maior parte dos recursos em todos os níveis de atenção. Os resultados não chegam a surpreender, visto que seguem o perfil da amostra. Isso não implica, porém, que não houve discriminação. Sem dúvida, uma análise antropológica capta melhor essa dimensão.

No cruzamento de informações sobre gênero (ou raça) e os quintis de renda, verificando os padrões de acesso para segmentos específicos da população (mulheres negras e de baixa renda, por exemplo), não se observou um padrão claro, por isso a análise não foi incorporada no estudo.

\section{NOTAS}

1. Muitos desses estudos têm sido realizados pelo Banco Mundial.

2. Bens não excludentes, ou seja, o consumo por parte de um indivíduo não exclui o consumo de outro indivíduo.

3. Na pesquisa que deu origem a este artigo também foi feita a aplicação do modelo no município de Cachoeira (BA).

4. Assim, considerou-se que pouco mais de $86 \%$ das transferências de média e alta complexidade (MAC) destinaram-se à atenção de média complexidade, e os $14 \%$ restantes destinaram-se à alta complexidade.

5. Esse mês foi escolhido em função de ser bem representativo da média anual.

6. Pesquisa de campo e Censo IBGE (2000).

\section{REFERÊNCIAS BIBLIOGRÁFICAS}

ALMEIDA FILHO, N. (1999) "Inequality in health base don living conditions: analysis of scientific output in latin America and the Caribbean and annotated bibliography". Pan American Health Organization, division of health and human development research coordination program, June. ELAC Project 19, 39 p.

ALMEIDA, C., TRAVASSOS, C., PORTO, S., LABRA, M. E. (2000) "Health sector reform in Brazil: a case study of inequity”. International Journal of Health Services, v. 30, n. 1.

BELTRÃO (2002) "Comparação de informações sobre saúde das populações brasileiras e norte-americana baseada em dados da PNAD/1998 e NHIS/1996”. Ciência e Saúde Coletiva, v. 7, n. 4 .

CASTRO-LeAl, F., DAYTON, J., DEMERY, L., MEHRA, K. (1999) "Public Social Spending in Africa: do the poor benefit"? In: Research Observer: The World Bank. v. 14, n. 1, February. p. 49-72. 
DACHS, J. N. (2002) “Determinantes das desigualdades na auto-avaliação do estado de saúde no Brasil: analise dos dados da PNAD/1998". Ciência e Saúde Coletiva, v. 7, n. 4, p. 641657.

(2001) "Inequidades en salud: como estudiarlas". In: Restrepo, $H$ \& Málaga, H. Promoción de la salud: como construir vida saludable. Editorial Médica Panamericana. Enero. $29 \mathrm{p}$.

DEMERY , L. (2000) "Benefit incidence: a practitioner's guide". Poverty and Social Development Group, Africa Region. The World Bank, Jul.

(1996) "Gender and Public Social Spending: disaggregating Benefit Incidence". Poverty and Social Development Group. The World Bank. May.

DEMERY, L. S. C. R. B., KALPANNA M. (1995) “The incidence of social Spending in Ghana, 1989-1992," The World Bank, draft.

DIAZ, M. D. M. (2001) “Desigualdades Socioeconômicas na Saúde no Brasil”. In: Texto para discussão n. 14. Programa de Seminários Acadêmicos, IPE/USP. São Paulo, p. 1-19.

(2002) “Gastos em saúde e incidência de benefícios no estado da Bahia” Consultoria para o Banco Mundial. Relatório Final. Washington, DC. jan.

FURTADO, C. (2004) “Água e esgoto para todos”. Desafios, ano 1, n. 5, p. 22-29, dez.

HOUSE, J., KESSELER, R. C., REGULA, H. A. (1990) “Age, socioeconomic status and health”. Milbank Quarterly, n. 3, p. 383-411.

IBGE (1998) “Pesquisa nacional de amostra de domicílios”. Instituto Brasileiro de Geografia e Estatística - IBGE - Cd-Rom.

IBGE, Censo (2000) Instituto Brasileiro de Geografia e Estatística - IBGE. Cd-Rom.

NERI, M., SOARES, W. (2002) “Desigualdade social e saúde no Brasil”. Caderno de Saúde Pública, Rio de Janeiro, n. 18 (Suplemento), p. 77-87.

NORONHA, K. V., VIEGAS, M. (2002a) “Desigualdades sociais em saúde: evidências empíricas sobre o caso brasileiro". Revista Econômica do Nordeste, n. 32 (Especial), p. 877-897.

NORONHA, K. V. M. S., ANDRADE, M. V. (2002b) “Desigualdade social no acesso aos serviços de saúde no Brasil” (mimeo).

OLIVEIRA, F. A. (1995) "Inter-relação e a interação classe, gênero e raça/etnia como elementos de análise da vida social”. (mimeo).

OLIVEIRA, F. (2003) “Saúde da população negra: Brasil ano 2001”. Brasília: Organização PanAmericana de saúde.

SAWYER, D. O., LEITE, I. DA C., ALEXANDRINO, R. (2002) “Perfis de utilização de serviços de saúde no Brasil". In: Acesso e uso de serviços de saúde no Brasil: uma análise da PNAD/98, v. 7, n. 4.

SILVA, L., PAIM, J., COSTA, M. (1999) "Desigualdades na Mortalidade por estratos sociais". Revista de Saúde Pública, v. 33, n. 2, p. 187-197.

TRAVASSOS, C., VIACAVA, F. FERNANDES, C., ALMEIDA, C. M. (2000) "Desigualdades geográficas e sociais na utilização de serviços de saúde no Brasil”. Ciência e Saúde Coletiva, Rio de Janeiro, v. 5, n. 1, jan. jul. 
TRAVASSOS, C., VIACAVA, F., PINHEIRO, R., BRITO, A. (2001) “Gênero e utilização de serviços de saúde no Brasil, Rio de Janeiro”. [S. 1.: s. n.]

VIANA, A. L., FAUSTO, M. C., LIMA, L. D. (2003) “Política de saúde e eqüidade”. São Paulo em Perspectiva, v. 17, n.1, p. 58-68.

VIANA, S. M. et al. (2001) "Medindo as desigualdades em saúde no Brasil: uma proposta de monitoramento: OPAS/IPEA”. Brasília.

WAGSTAFF, A. (2001) "Poverty and Health". CMH Working Paper Series. Paper, n. Wg1:5, p. 52, March.

WOOD, C., CARVALHO, J. (1994) Desigualdade de renda e expectativa de vida. Rio de Janeiro: PNPE/IPEA. 